\title{
Ocular Surface Diseases Induced by Dupilumab in Severe Atopic Dermatitis
}

\author{
Adrien Maudinet - Sandrine Law-Koune - Claire Duretz • \\ Audrey Lasek · Philippe Modiano · Thi Ha Chau Tran
}

Received: April 9, 2019 / Published online: June 22, 2019

(c) The Author(s) 2019

\begin{abstract}
We report 10 cases of conjunctivitis in atopic dermatitis (AD) patients treated with dupilumab from November 2017 to November 2018 in our institution, who were referred to the ophthalmology department for diagnosis and management of conjunctivitis. We also describe ocular surface findings in these patients before the first injection of dupilumab. During the first 6 months post initiation of dupilumab, incidence of conjunctivitis was $27 \%(5 / 18)$ in patients treated from November 2017 to April 2018 who had not had ocular examination previously. This rate dropped to $12 \%(3 / 25)$
\end{abstract}

Enhanced digital features To view enhanced digital features for this article go to https://doi.org/10.6084/ m9.figshare.8209520.

Electronic supplementary material The online version of this article (https://doi.org/10.1007/s40123019-0191-9) contains supplementary material, which is available to authorized users.

A. Maudinet · S. Law-Koune

Ophthalmology Department, Lille Catholic

Hospitals, Lille Catholic University, Lille, France

C. Duretz · A. Lasek · P. Modiano

Dermatology Department, Lille Catholic Hospitals, Lille Catholic University, Lille, France

T. H. C. Tran $(\bowtie)$

Ophthalmology Department, Saint-Vincent de Paul Hospital, Boulevard de Belfort, Lille Cedex, France e-mail: tran.hachau@ghicl.net after systematic ophthalmological referral before initiation of dupilumab. Patients who developed conjunctivitis had mean SCORAD score (Scoring Atopic Dermatitis) of $60.4 \pm 20$ (35-88) and mean EASI score (Eczema Area and Severity Index) of $37 \pm 17$ (14.6-56). Mean age was 36 years (20-51). Most patients had a long history of $\mathrm{AD}$ ( $>10$ years). Mean delay of ocular surface inflammation was 3.5 months, ranging from 1 to 8 months. One patient had to discontinue dupilumab because of severe follicular conjunctivitis. We observed two clinical patterns of ocular surface diseases: a mild nonspecific conjunctivitis with dry eyes, which improved with warm compresses and artificial tears without any recurrence; and a severe dupilumab-induced follicular conjunctivitis without keratitis, which required specific ophthalmological management.

Keywords: Atopic dermatitis; Conjunctivitis; Dry eye; Dupilumab; Iatrogenic ocular surface disease; Keratitis

\section{INTRODUCTION}

Dupilumab blocks signaling of interleukin 4 and interleukin 13, Th2 cytokines implicated in allergic diseases such as asthma and atopic dermatitis. Dupilumab received Food Drug Administration (FDA) approval for moderate 
and severe atopic dermatitis (AD) in March 2017, and European Medicines Agency (EMA) authorization in September 2017. Higher rates of conjunctivitis have been reported in phase 3 clinical trials in patients treated with dupilumab for atopic dermatitis (14-19\%), compared to the placebo group (8\%) [1-3]. However, conjunctivitis was reported by investigators without any additional workup and ophthalmological referral. In clinical trials where these cases resolved with topical eye drops prescribed by dermatologists, only one of 425 treated patients discontinued dupilumab because of conjunctivitis $[1,4]$. However, the incidence of ocular surface diseases induced by dupilumab seems to be higher in real life than pivotal study, up to $23 \%$ [5]. Here we report our experience of conjunctivitis in $\mathrm{AD}$ patients treated with dupilumab since November 2017 in real life in our department, who were referred by the dermatology department for diagnosis and management of conjunctivitis. We also report ocular surface findings in these patients before the first injection of dupilumab.

The Institutional Review Board of Lille Catholic Hospitals approved this case series. All subjects provided informed consent prior to participation and publication of this case series. Consent was obtained for publication of Fig. 1.

\section{CASES PRESENTATION OF OCULAR SURFACE DISEASES IN SEVERE ATOPIC DERMATITIS PATIENTS TREATED WITH DUPILUMAB}

\section{Baseline Ophthalmologic Examination and the Incidence of Ocular Surface Disease Under Dupilumab Therapy}

In our center, incidence of conjunctivitis was $5 / 18(27 \%)$ during the first 6 months post initiation of dupilumab, in patients treated from November 2017 to April 2018, when patients were not referred to an ophthalmologist before the first injection. From May to November 2018, 25 patients underwent ophthalmologic examination systematically before treatment. At baseline, most of them $(16 / 25,64 \%)$ had abnormal ocular surface including posterior blepharitis $(14 / 25)$, associated or not with mild conjunctivitis $(4 / 25)$, corneal epithelium lesion $(6 / 25)$, and limbal neovascularization $(1 / 25)$. Management of ocular surface disease of the AD patients included warm compresses (58\%), artificial tears (25\%), and antihistamine drops $(8 \%)$. With this preventive management, conjunctivitis incidence dropped to $3 / 25$ (12\%) after at least 3 months of follow-up.

\section{Clinical Characteristics of Ocular Surface Disease Under Dupilumab Therapy}

Clinical characteristics of ocular surface disease of $10 \mathrm{AD}$ patients treated with dupilumab therapy are summarized in Table $\mathrm{S} 1$ (see supplementary material). Mean age was 36 years (20-51). Most patients had a long history of AD (>10 years). Mean SCORAD (Scoring Atopic Dermatitis) score was $60.4 \pm 20(35-88)$ and mean EASI score (Eczema Area and Severity Index) was $37 \pm 17$ (14.6-56). Eczema area included head/neck and body localization in $7 / 10$ patients, and only the body in three patients. All had comorbid type 2 immune diseases: food or other allergy (8/10) and asthma $(8 / 10)$. One patient had previously undergone steroid-induced cataract surgery. History of allergic conjunctivitis was found in $6 / 10$ patients. Mean delay of ocular surface inflammation was 3.5 months, ranging from 1 to 8 months. One patient (patient 2) had to discontinue dupilumab 1 month after development of follicular conjunctivitis.

Among these 10 patients, ophthalmologic evaluation was performed in three patients (patient 8, 9, and 10) before initiation of dupilumab. Patients 7 and 8 had blepharitis and allergic keratoconjunctivitis which improved with warms compresses, artificial tears, and antihistamine drops. They presented exacerbation of ocular surface inflammation 4-6 months later with different clinical presentation. Patient 10 had normal ocular surface before dupilumab injection. In seven other patients, ocular evaluation was lacking prior to dupilumab injection. Among these, prednisolone drops were given in three patients by a general practitioner 

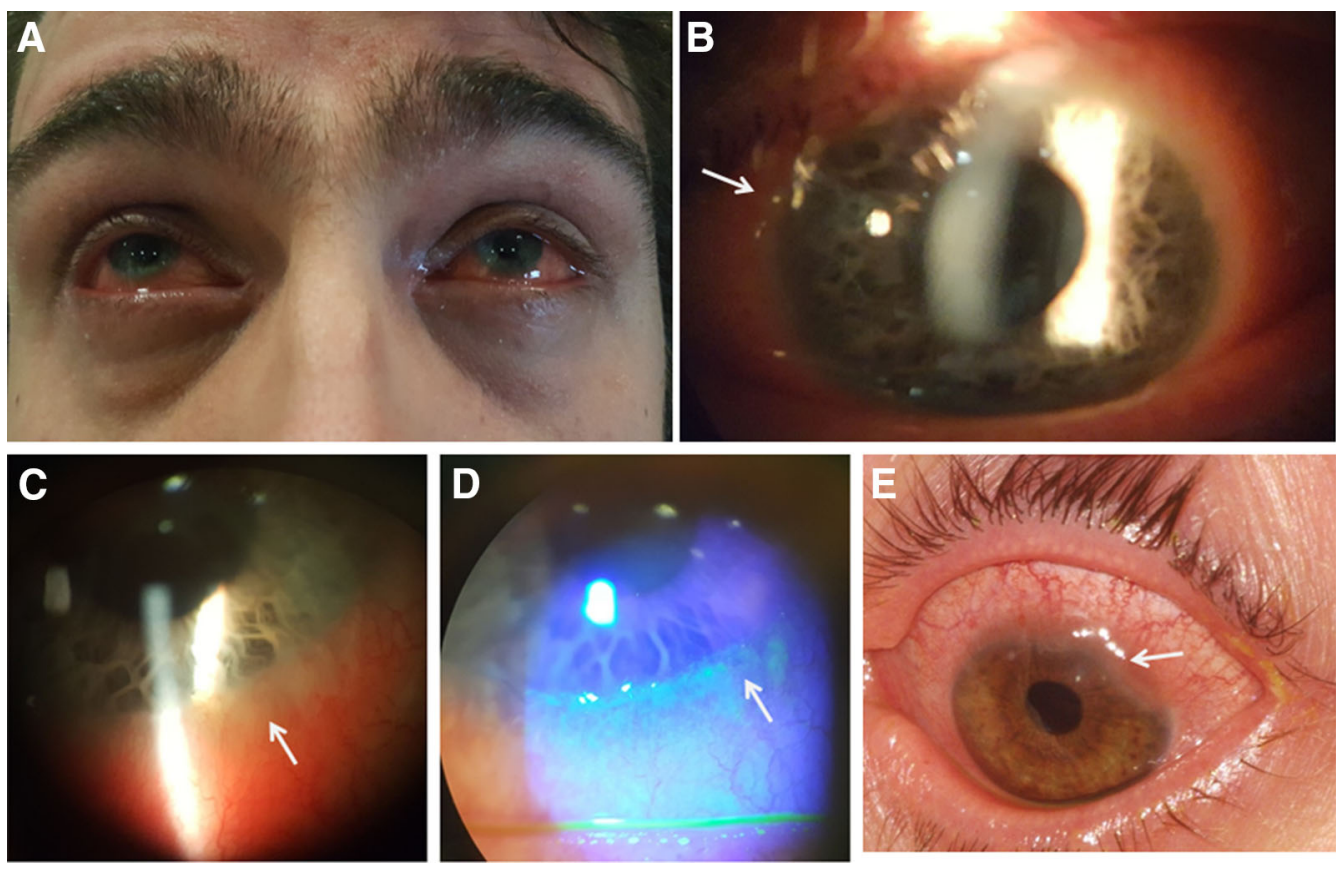

Fig. 1 Severe tearing and hyperemia of bulbar and tarsal conjunctiva (a) and perilimbal nodules (arrow) in patient 2 (b), in patient 8 (c, d), and limbitis in patient $9(\mathbf{e})$

or dermatologist without any effectiveness. In most patients, typical symptoms included itching, stinging, burning, tearing, and photophobia in both eyes. We observed two clinical patterns of ocular surface diseases.

\section{Non-specific Conjunctivitis Associated with Inferior Punctuated Corneal Epithelial Lesion}

Six patients (1, 3, 4, 5, 7, and 10) had mild conjunctivitis associated with inferior punctuated corneal epithelial lesion. They had no aqueous deficiency but evaporative dry eyes with TBUT (Tear Film Beak up Time) less than 10 s. Patients 4 and 5 did not respond to dexamethasone drops before referral. Therapy included application of warm compresses and rehydration of ocular surface using non-preserved formulations as high viscosity artificial tears which were then switched to sodium hyaluronate, then to trehalose/hyaluronate tear substitute. They all improved with this treatment without any recurrence.

\section{Severe Dupilumab-Induced Follicular Conjunctivitis Without Keratitis}

Four patients had follicular conjunctivitis $(2,6$, 8 , and 9) which occurred from 3 to 6 months after dupilumab initiation. All patients reported improvement of atopic dermatitis. Principal ocular symptoms were tearing, photophobia, and intermittent blurred vision leading to limiting daily activities. Three of these four patients had history of allergic conjunctivitis which occurred or worsened after onset of dupilumab. All of them had blepharitis, moderate to severe hyperemia of the conjunctiva, and eyelid margins which extend beyond the interpalpebral zone. There was no aqueous tear deficiency and no evaporative deficiency. Nodular swelling of the limbus was found in both eyes (Figs. 1, 2) resembling Trantas dots, but they were located more anteriorly on the limbus. No damage of the corneal epithelium was found. The follicular conjunctivitis occurred even if patients were treated with artificial tears and antihistamine eye drops (ketotifen) for allergic conjunctivitis and dry eyes. In patients 6,8 , and 9 , conjunctivitis improved significantly in a few days with dexamethasone eye drops which were then 

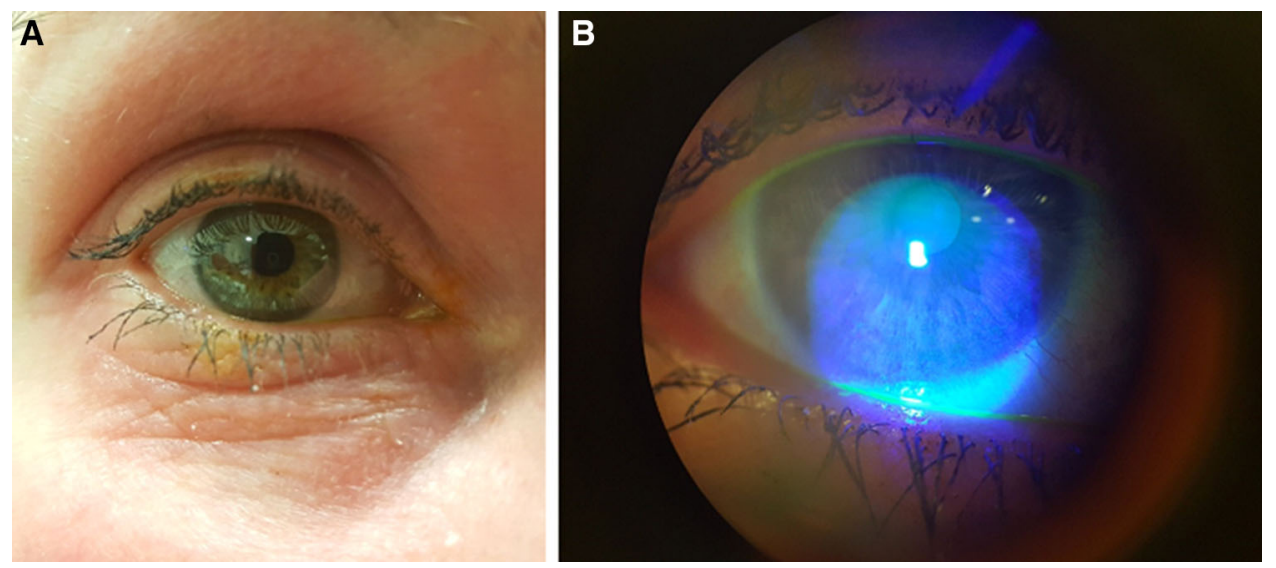

Fig. 2 Mild periocular dermatitis and mild conjunctivitis (a). Non-specific inferior corneal epithelial punctuate keratitis (b)

tapered within a few weeks, associated with unpreserved trehalose/hyaluronate tear substitute without recurrence after 3 months of follow-up. Patient 2 suffered from severe conjunctivitis which did not improve through dexamethasone + trehalose/hyaluronate tear substitute + cyclosporine $1 \%$. Reduced frequency followed by discontinuation of dupilumab was necessary, and conjunctivitis improved slowly 2 months later.

\section{DISCUSSION}

Clinical characteristics of AD in this small series are comparable to those of clinical trials [3]. In the LIBERTY AD CAFÉ study, which included severe $\mathrm{AD}$ with an inadequate response or intolerance to cyclosporine, incidence of conjunctivitis was up to $28 \%$ in dupilumab-treated patients [4]. The incidence of conjunctivitis confirmed by ophthalmologists in real life seems to be greater, namely $25 \%$ in our center and in $23 \%$ in another report [5].

We summarized dupilumab-induced ocular surface disease reported in the literature in Table S2 (supplementary material). We also found two types of dupilumab-induced ocular surface disease: a mild non-specific conjunctivitis and keratitis with dry eyes and a more specific dupilumab-induced follicular conjunctivitis and limbitis. Most cases were mild or moderate [6]. Most of the patients did not have any history of conjunctivitis. This conjunctivitis was associated with blepharitis in $50-77 \%$ of cases or punctual stenosis leading to severe tearing and ectropion in some cases [5-9]. The follicular conjunctivitis occurred despite antihistamine and hyaluronic acid drops as previously described [6]. Treatment of this follicular conjunctivitis depends on the severity of inflammation, varying from local steroid (fluorometholone 1\%, dexamethasone $0.1 \%$, prednisolone) which was tapered from 2 to 4 weeks, associated with tacrolimus eye ointment $0.03 \%$ or cyclosporine eye drops [6]. In rare cases $(0.2-0.4 \%)$, ocular inflammation did not respond to any drops, requiring discontinuation of dupilumab therapy $[7,10,11]$. Blepharoconjunctivitis may be responsible for subepithelial fibrosis, suggesting a potential severity if inflammation is not controlled [12].

In our experience, $64 \%$ of DA patients had abnormal ocular surface with posterior blepharitis, evaporative dry eyes, conjunctivitis, keratitis, and limbal neovascularization without any ocular complaints at baseline. We would suggest referral at initiation of dupilumab to classify ocular surface inflammation, preventive treatment of meibomian gland dysfunction, moisture of ocular surface with unpreserved artificial tears, and referral to an ophthalmologist for evaluation and appropriate treatment if follicular conjunctivitis occurred.

The underlying mechanism for the development of conjunctivitis seems to be inflammatory and specific to AD since this side effect was not noted in patients with asthma or nasal 
polyposis on dupilumab [13]. Frustratingly, severe conjunctivitis occurred in $\mathrm{AD}$ patients who responded to dupilumab in our series [10].

Histological findings in conjunctivitis induced by dupilumab reported by Bakker et al. [5] revealed focal scarcity of intra-epithelial goblet cells, which is different from the histopathology of allergic conjunctivitis. This explains mucin deficiency which affects the stability of the tear film. We used a combination of trehalose/hyaluronate since it has effects on ocular surface inflammatory markers, mucin expression (decrease in IL-1 $\beta$, IL-6, and IL-8 tear levels), and goblet cell density recovery, targeting the mechanism of dupilumab-induced ocular surface disease [5]. Topical cyclosporine eye drops could be combined with dupilumab treatment in case of severe dermatitis. In fact, cyclosporine, as suggested by Shen et al., is a T cell-mediated immune response via decreased expression of IL- 4 for the follicular conjunctivitis. Both tacrolimus and cyclosporine are ophthalmic calcineurin inhibitors that inhibit T cell-mediated immune response [8]. While steroid drops can cause serious side effects (infections, glaucoma, or cataracts), cyclosporine does not enter the anterior chamber and has good immunomodulatory effect on the ocular surface. It can also be administered four times a day at $2 \%$ as a low-cost galenic product for the patients. Its only side effect is a burning sensation which occurs occasionally on the application of eye drops. By suppressing inflammation, cyclosporine is also considered capable of increasing conjunctival goblet cell density and decreasing corneal cells apoptosis. The therapeutic effects of cyclosporine drops in the treatment of eyes affected by several keratoconjunctivitis have been proven in numerous studies in children and adults [14, 15].

Dupilumab blocks signaling both IL-4 and IL-13, which are Th2-mediated inflammation cytokines. Consequently, the upregulation leading to an increase of Th1 response and IFN$\gamma$-mediated inflammation is also a possible mechanism. The pro-inflammatory IFN- $\gamma$ has been proven as a biomarker for evaporative dry eye disease [16] and cell-mediated immune response in the mucosal immune compartment leading to a variety of dupilumab-induced inflammation spectrum: blepharitis, tear film instability, meibomian gland dysfunction, goblet cell deficiency, exposure keratopathy, follicular conjunctivitis and limbitis, punctual stenosis, and periocular dermatitis.

Further elucidation of dupilumab's immunologic effect of the tear film is needed to better understand the underlying mechanism of the ocular and periocular side effects and the Th1/Th2 paradigm in ocular allergy.

\section{ACKNOWLEDGEMENTS}

Funding. No funding or sponsorship was received for this study or publication of this article.

Editorial assistance. The authors gratefully acknowledged the English editorial assistance of Ms. Nhi Van for the paper. No funding was received for this assistance.

Authorship. All named authors meet the International Committee of Medical Journal Editors (ICMJE) criteria for authorship for this article, take responsibility for the integrity of the work as a whole, and have given their approval for this version to be published.

Disclosures. Adrien Maudinet, Sandrine Law-Koune, Claire Duretz, Audrey Lasek, Philippe Modiano, and Thi Ha Chau Tran have nothing to disclose.

Compliance with ethics guidelines. The Institutional Review Board of Lille Catholic Hospitals approved this case series. All subjects provided informed consent prior to participation and publication of this case series. Consent was obtained for publication of Fig. 1.

Open Access. This article is distributed under the terms of the Creative Commons Attribution-NonCommercial 4.0 International License (http://creativecommons.org/licenses/ by-nc/4.0/), which permits any noncommercial use, distribution, and reproduction in any medium, provided you give appropriate credit 
to the original author(s) and the source, provide a link to the Creative Commons license, and indicate if changes were made.

\section{REFERENCES}

1. Blauvelt A, de Bruin-Weller M, Gooderham M, et al. Long-term management of moderate-to-severe atopic dermatitis with dupilumab and concomitant topical corticosteroids (LIBERTY AD CHRONOS): a 1-year, randomised, double-blinded, placebo-controlled, phase 3 trial. Lancet. 2017;389(10086):2287-303.

2. Ou Z, Chen C, Chen A, Yang Y, Zhou W. Adverse events of dupilumab in adults with moderate-tosevere atopic dermatitis: a meta-analysis. Int Immunopharmacol. 2018;54:303-10.

3. Simpson EL, Bieber T, Guttman-Yassky E, et al. Two phase 3 trials of dupilumab versus placebo in atopic dermatitis. N Engl J Med. 2016;375(24):2335-48.

4. de Bruin-Weller $\mathrm{M}$, Thaci $\mathrm{D}$, Smith $\mathrm{CH}$, et al. Dupilumab with concomitant topical corticosteroid treatment in adults with atopic dermatitis with an inadequate response or intolerance to ciclosporin $\mathrm{A}$ or when this treatment is medically inadvisable: a placebo-controlled, randomized phase III clinical trial (LIBERTY AD CAFE). $\mathrm{Br} J$ Dermatol. 2018;178(5):1083-101.

5. Bakker DS, Ariens LFM, van Luijk C, et al. Goblet cell scarcity and conjunctival inflammation during treatment with dupilumab in patients with atopic dermatitis. Br J Dermatol. 2019;180(5):1248-1249.

6. Wollenberg A, Ariens L, Thurau S, van Luijk C, Seegraber M, de Bruin-Weller M. Conjunctivitis occurring in atopic dermatitis patients treated with dupilumab-clinical characteristics and treatment. J Allergy Clin Immunol Pract. 2018;6(5):1778-1780.e1.

7. Barnes AC, Blandford AD, Perry JD. Cicatricial ectropion in a patient treated with dupilumab. Am J Ophthalmol Case Rep. 2017;7:120-2.
8. Shen E, Xie K, Jwo K, Smith J, Mosaed S. Dupilumab-induced follicular conjunctivitis. Ocular Immunol Inflamm. 2018;1-3. https://doi.org/10. $1080 / 09273948.2018 .1533567$.

9. Zirwas MJ, Wulff K, Beckman K. Lifitegrast add-on treatment for dupilumab-induced ocular surface disease (DIOSD): a novel case report. JAAD Case Rep. 2019;5(1):34-6.

10. Treister AD, Kraff-Cooper C, Lio PA. Risk factors for dupilumab-associated conjunctivitis in patients with atopic dermatitis. JAMA Dermatol. 2018;154(10):1208-11.

11. Yamane MLM, Belsito DV, Glass LRD. Two differing presentations of periocular dermatitis as a side effect of dupilumab for atopic dermatitis. Orbit. 2019;1-5. https://doi.org/10.1080/01676830.2018. 1553190 .

12. Levine RM, Tattersall IW, Gaudio PA, King BA. Cicatrizing blepharoconjunctivitis occurring during dupilumab treatment and a proposed algorithm for its management. JAMA Dermatol. 2018;154(12):1485-6.

13. Rabe KF, Nair P, Brusselle G, et al. Efficacy and safety of dupilumab in glucocorticoid-dependent severe asthma. N Engl J Med. 2018;378(26):2475-85.

14. Nebbioso M, Zicari AM, Celani C, Lollobrigida V, Grenga R, Duse M. Pathogenesis of vernal keratoconjunctivitis and associated factors. Semin Ophthalmol. 2015;30(5-6):340-4.

15. Leonardi A, Doan S, Amrane M, et al. A randomized, controlled trial of cyclosporine a cationic emulsion in pediatric vernal keratoconjunctivitis: the VEKTIS Study. Ophthalmology. 2019;126(5):671-81.

16. Jackson DC, Zeng W, Wong CY, et al. Tear interferon-gamma as a biomarker for evaporative dry eye disease. Invest Ophthalmol Vis Sci. 2016;57(11):4824-30. 